\title{
Capsule Commentary on VanGompel et al., Incidence and Predictors of Repeat Bone Mineral Densitometry: A Longitudinal Cohort Study
}

\author{
Kay M. Johnson, MD, MPH ${ }^{1,2}$ \\ 'Division of General Internal Medicine, University of Washington School of Medicine, Seattle, WA, USA; ${ }^{2}$ VA Puget Sound Health Care System, \\ Seattle, WA, USA.
}

$\mathrm{J}$ Gen Intern Med 32(10): 1131

DOI: $10.1007 / \mathrm{s} 11606-017-4132-9$

(c) Society of General Internal Medicine (outside the USA) 2017

$\mathrm{T}$ he U.S. Preventive Services Task Force (USPSTF) recommends dual x-ray absorptiometry (DXA) scans in women 65 years of age and older, and in younger women with an increased fracture risk. ${ }^{1}$ VanGompel and colleagues studied physicians' use of repeat DXA among 6000 women aged 4084 who received initial DXA screening within a regional health system in California. ${ }^{2}$ The vast majority $(72 \%)$ were younger than 65 . Osteoporosis was found in $14 \%$ of women; $13 \%$ had a high risk of progression to osteoporosis, while most (74\%) had a low risk of progression. An osteoporosis drug was prescribed for only $68.9 \%$ of those with osteoporosis, but was given to $19.1 \%$ of women at low risk. Among those untreated, only $60 \%$ of high-risk women had follow-up scans within 5 years, though $43 \%$ of women at low risk of progression were scanned. Among treated women, median time to repeat DXA was 3 years. Patients in both the treated and untreated groups who were followed by an endocrinologist were more likely to have repeat DXA.

What is disturbing is that only $60 \%$ of the untreated highrisk women received a follow-up DXA within 5 years; by this time, half of these women would have already transitioned to osteoporosis. $^{3}$ And since only $10 \%$ of women with normal density or mild osteopenia will transition to osteoporosis in the ensuing 16 years, ${ }^{3}$ many women in the low-risk group were rescreened too soon.

Important limitations include the source of data from a single health care system, the use of a complicated definition of low vs. high risk which differs somewhat from national guidelines, and exclusion of $40 \%$ of scans due to incomplete T-score data.

Based on this study, and in light of recent literature, resource allocation can be improved by limiting DXA screening to women who meet USPSTF guidelines, focusing on those who would consider osteoporosis treatment if positive, and by avoiding a repeat DXA within 15 years for women with low risk of progression. Ways to improve osteoporosis treatment include treating a higher percentage of women who screen positive, and either treating women who are at high risk of progression ${ }^{4,5}$ or re-screening them in 2 years.

Corresponding Author: Kay M. Johnson, MD, MPH; VA Puget Sound Health Care System, Seattle, WA, USA (e-mail: Kay.Johnson2@va. gov).

\section{Compliance with Ethical Standards:}

Conflict of Interest: The author has no conflicts of interest with this article.

\section{REFERENCES}

1. U.S. Preventive Services Task Force. Screening for osteoporosis: U.S. Preventive Services Task Force Recommendation Statement. Ann Intern Med. 2011 Mar 1;154(5):356-64.

2. VanGompel ECW, Franks P, Robbins JA, Fenton JF. Incidence and predictors of repeat bone mineral densitometry: a longitudinal cohort study. J Gen Intern Med. doi:10.1007/s11606-017-4094-y

3. Gourlay ML, Fine JP, Preisser JS, et al. Bone-density testing interval and transition to osteoporosis in older women. New Engl J Med. 2012;366(3):225-233.

4. Cosman F, de Beur SJ, LeBoff MS, et al. Clinician's Guide to Prevention and Treatment of Osteoporosis. Osteoporos Int. 2014 Oct;25(10):2359-81.

5. World Health Organization. Fracture Risk Assessment Tool (FRAX). Available at: https://www.shef.ac.uk/FRAX/. Accessed June 30, 2017. 